\title{
The Design and Application of College English-Aided Teaching System Based on Web
}

\author{
Rui Chen \\ Chongqing Vocational College of Applied Technology, Chongqing 401520, China \\ Correspondence should be addressed to Rui Chen; 201009157069@wust.edu.cn
}

Received 12 October 2021; Accepted 3 January 2022; Published 25 February 2022

Academic Editor: Mukhtaj khan

Copyright ( $\odot 2022$ Rui Chen. This is an open access article distributed under the Creative Commons Attribution License, which permits unrestricted use, distribution, and reproduction in any medium, provided the original work is properly cited.

\begin{abstract}
With the rapid advancement of information technology, the Internet and the web have become the central technology tools for information sharing in education and teaching. Modern education combines teaching with information technology and gives full play to the role of information technology in education and teaching. To improve the resource sharing and scheduling level a webbased college English-assisted teaching system is presented in this study. Using the JavaScript object notation syntax (JSON) as the data communication format between the intelligent terminal and the server, the system is implemented using a web server and mobile Android terminal, and a web-based data integration module is designed. Statistical data mining and fuzzy feature detection are employed to implement the decision-making scheduling and self-adaptive optimization of English teaching resources in a web aided English teaching system. The fusion particle swarm optimization algorithm is used to integrate English teaching resources and implement adaptive scheduling. To verify the application performance of the designed system, a simulation experiment is designed. The experimental results show that the proposed English-assisted instruction system has good convergence and high accuracy of learning information, optimal allocation, and scheduling.
\end{abstract}

\section{Introduction}

The development of computer and networking technologies has led to the continuous innovation and advancement of online education methods [1]. Teachers and students can teach and learn anywhere and anytime without the restriction of space and time. Due to this accessibility of network teaching, many teachers and students prefer the network teaching process, making network teaching a new platform of education and learning. With the features of digitalization, interconnectivity, huge multimedia information, and wide range, it allows more learners to receive a good education and contributes to the equity of education [2]. However, there are still some limitations in the real online teaching and evaluation environment, which greatly hinder and restrict the online teaching and evaluation process.

The evaluation of online teaching is also different from traditional teaching, which mostly gives evaluation results through expert advice, which is subjective and not very objective. The traditional evaluation method involves collecting various teaching-related contents and material investment, wasting a lot of time and costs and creating too much overhead. Moreover, most of the traditional teaching evaluation methods use the evaluation of students and teachers based on their performance at the end or beginning of the session. As far as the development of teaching evaluation is concerned, establishing a fair and transparent teaching evaluation system is the top priority of teaching evaluation. It is related to not only the improvement of teaching quality, but also the scientific and standardization of teaching evaluation work. The convenience of online teaching makes teachers and students attend classes at irregular times and locations, which makes teaching convenient.

Chen et al. [3] developed an intelligent teaching system based on in-depth learning. The system was comprised of two parts: online personalized learning recommendation and offline classroom quality two-way evaluation. In the online system, performance prediction and online learning behavior law 
analysis based on in-depth learning were designed, and learning emotion classification is realized combined with image processing techniques. In the offline system, using target detection, face recognition models, personalized learning recommendations are realized. At the same time, the evaluation and feedback of college teaching quality and students' learning behavior are employed using offline classroom information data. An information-based teaching management system integrating association rule mining algorithms is presented in [4]. The key components of the system are client, server, and database. The client and server were, respectively, responsible for the presentation of user-oriented interactive functions and the logical operation of system applications. The system creates a database system covering different aspects of teaching management and adopts an improved association rule mining algorithm, mines the related data of users' educational information in all aspects from the system database, and provides users with teaching, course selection, course, and other services through the client main interface. Zhao and Guo [5] proposed a web-based teaching and evaluation system using campus network resources. The system was effective in promoting communication among teachers and students. The system was comprised of three-layer architecture and provided administrator management, exam settings, test questions, teaching evaluation, and students' learning functions. Meng et al. [6] designed an intelligent system of English culture teaching and elaborated its four aspects of personalization, virtualization, intelligentization, and quality orientation of cultural learning and teaching. Aparicio et al. [7] proposed intelligent information access systems to evaluate the teachers' perceptions concerning the utility of these systems in learning activities. The system was employed to realize its applicability in activities in both the preparation and the effective use of these activities in the classroom. Lu [8] demonstrated artificial intelligence-based writing evaluation systems such as AWE for reducing the English teachers' workload and keeping up with the students' writing level.

College English teaching is a part of college humanistic education and is one of the vital sources to promote crosscultural communication aptitudes. However, there are still some problems in college English education, such as lacking comparative study, scanty high-quality teaching resources, suitable textbooks, and outdated teaching methods. Therefore, designing a college English-assisted instruction system is of great significance to promote the development and construction of higher education information.

To improve the resource sharing and scheduling level a web-based college English-assisted teaching system is presented in this study. Using JSON as the data communication format between the intelligent mobile Android terminal and the server, the system is implemented by using mobile Android terminal and web, and a web-based data integration module is designed. Through the fusion of particle swarm optimization, the adaptive scheduling of English teaching resources in colleges and universities is realized. The experimental results show that the proposed system has better convergence, and the allocation and scheduling of English teaching resources are more effective and practical.
The rest of the paper is ordered as follows: in Section 2 the architecture of the proposed system and its different modules are discussed. In Section 3 the experimental setup of the proposed system and its validation are presented. Finally the conclusion is given in Section 4.

\section{Design of College English-Aided Teaching System}

The web-based online English course-aided teaching system is implemented with the help of a web server and Android mobile terminal. The main function of the online interactive part is to provide the channel of teaching interaction and enable sharing of teaching resources among teachers and students [9]. The overall framework of the proposed college English-aided teaching system is shown in Figure 1.

\subsection{Hardware Design}

2.1.1. Android Server and Client Design. JSON is adopted as the data exchange format for the intelligent terminal to communicate with the server [10]. The PC equipped with the interactive system is not only the application server for accessing the browser but also the Android server for responding to the requests and feedback of the Android client.

As an important server of the online interaction module, the communication with student Android is also realized by it, and the communication service is for user login verification and information promotion. The Android accesses the server-side after connecting to the intranet of the classroom and communicates according to custom JSON format data. The communication flow between the server and the client is shown in Figure 2.

It can be seen that the framework of the server is MVC architecture mode, which is divided into three parts: model, view, and controller. The structure of the MVC model is shown in Figure 3.

The model section includes data and business logic models, which are used to store data and run operations. The view part is a content presentation, which executes sending request instruction when the user operates and the controller part shows the processing results on the view by expanding the business logic judgment on the received request.

Because there are situations where students need teachers to take the initiative to send messages to students without initiating a request when interacting online, the MQTT (Message Queuing Telemetry Transport, Message Queue Telemetry Transmission) communication protocol [11], developed by IBM, is used to deliver messages, which is an instant messaging protocol suitable for limited computing capacity and unreliable network environments, especially unstable networks for mobile phones. The message push flow for this communication protocol is shown in Figure 4.

The server uses the message proxy of the MQTT protocol to push the message to the Android client, sends 


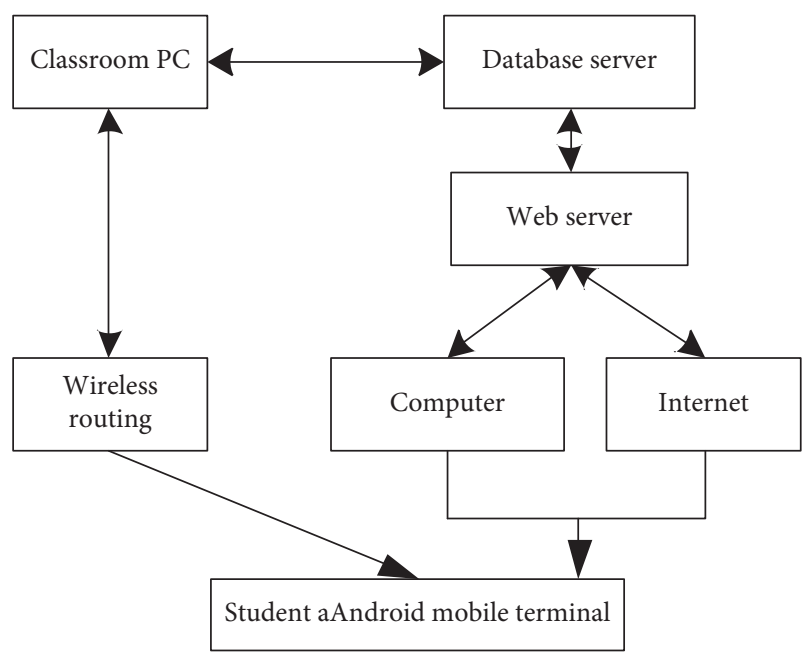

FIGURE 1: College English-aided teaching system.

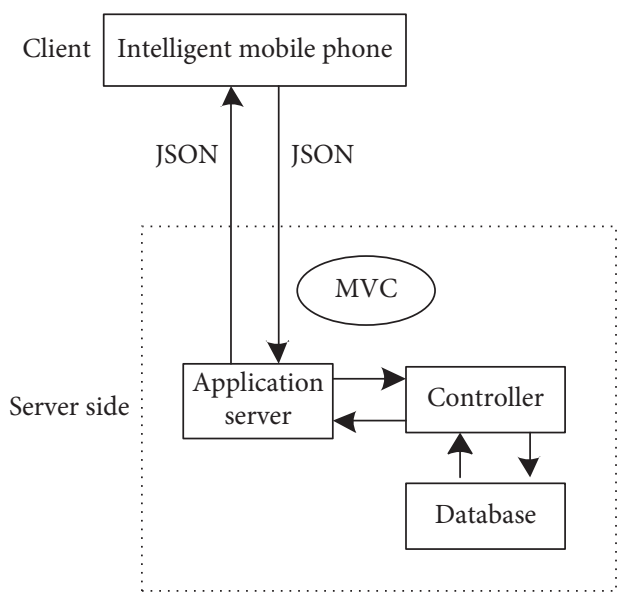

Figure 2: Processing framework between server and client.

the message to the message proxy, selects the message receiving target based on the protocol rules, completes the message push of the mobile client, and achieves online interaction.

To meet the needs of the college English course teaching system, teachers use the server to interact with the students, such as checking the students' attendance, pushing the questions, and supervising the learning. The teacher-side interaction module is composed of multiple parts as shown in Figure 5.

The client is mainly employed to provide online interaction between students and teachers, which is composed of three functional modules: scanning two-dimensional code, classroom interaction, and offline login. After scanning the server-side QR code, the student logs in and signs in and receives an instant message from the teacher or replies to it. If there is no intranet, the student logs in offline to view and review the historical information sent by the teacher. Using the online learning interface, students can connect to the external network for autonomous learning operations. The student interaction module consists of multiple functions as shown in Figure 6.
2.1.2. Web Server-Side Design. Based on the web mode, the web server side is constructed using components such as browser, data block service, and web service. The logical framework is shown in Figure 7.

After the web server receives the HTTP request from the client browser, it queries the database. When the browser receives the query result, it will automatically parse JS, CSS, and other files [12]. The role of a database server is to store data and provide data access services. The web server is integrated with an integrated $\mathrm{SSH}$ framework of spring, struts 2, and hibernate technologies as shown in Figure 8. Among them, the business logic layer deals with business planning, data access, authentication, and other tasks; the client uses the component to connect the layer and complete its interaction with the database.

To reduce the storage space and facilitate network transmission, the system uses the FFmpeg multimedia codec framework to automatically convert the video coding uploaded by the teacher. The bootstrap front-end development framework based on CSS, HTML, and JAVASCRIPT is used in the coding design process to facilitate students' access to online learning modules.

The learning modules provide different functions for different target groups and are categorized into task execution and online learning. Students will be prompted to complete the number of tasks after successfully logging in to the system, and the task can be carried out after selecting the number of tasks. In addition to the above functions, the module also supports information and password modification.

2.1.3. Design of Interactive Integration Module of College English Teaching System. With the continuous development of cloud computing technology on the Internet, the service architecture in the system server will become more and more complex [13]. The data expansion capabilities of the single Internet server architecture are limited, and the failure of a single module in the server will affect the operation of the entire system. Therefore, this study designs a web interface server architecture with a multiarchitecture mod that can effectively face the complexity and diversity of English teaching data. In this study, the intensive input-output data storage container is adopted as the data expansion storage main body of the server. This type of data storage container has an asynchronous programming chip inside, which can timely process the data content of multistep teaching. This container has small space possession and high flexibility and can be disassembled and replaced. The way of data expansion is mainly through the web interface of college English data debugging. When the data capacity of other data expansion containers is insufficient, the data storage containers in other machines' equipment of the proposed system can be adjusted and managed through the web interface to balance the storage data load. Figure 9 shows the hardware system architecture of the proposed college English-aided system.

The server model used in the present study is the Core I5-8530 series by Intel. The processing frequency of the data 


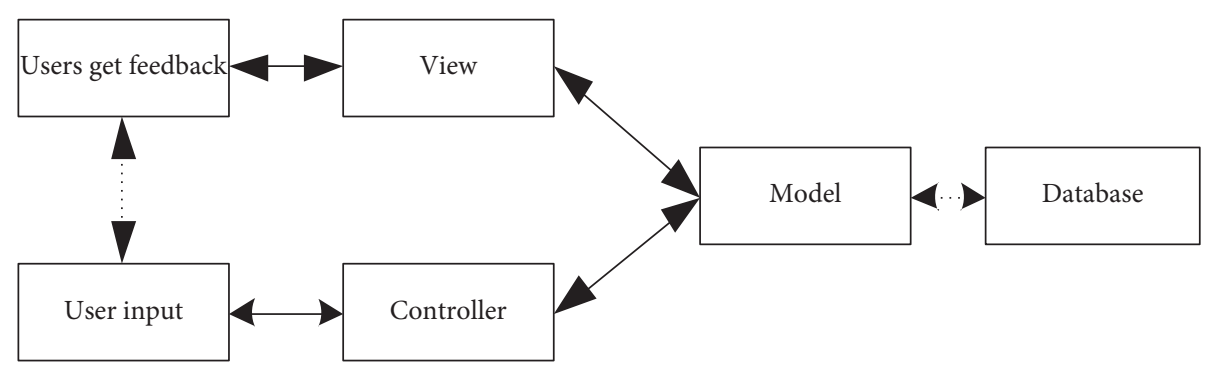

FIgURe 3: MVC structure.

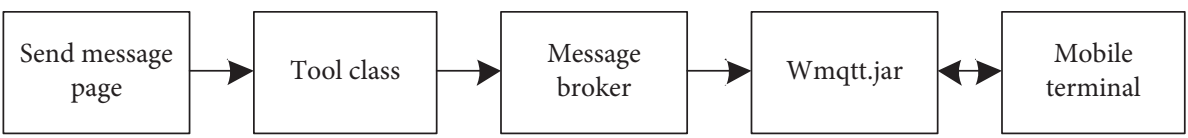

FIGURE 4: Schematic diagram of message push process.

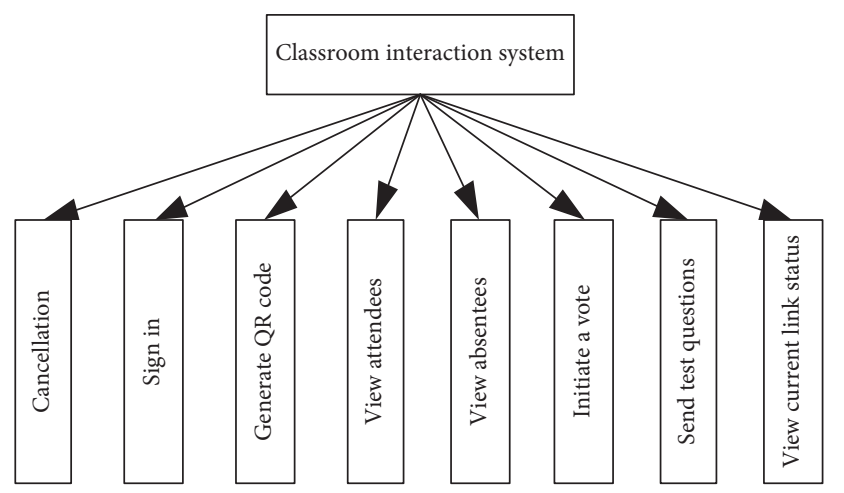

Figure 5: Teacher interaction module diagram.

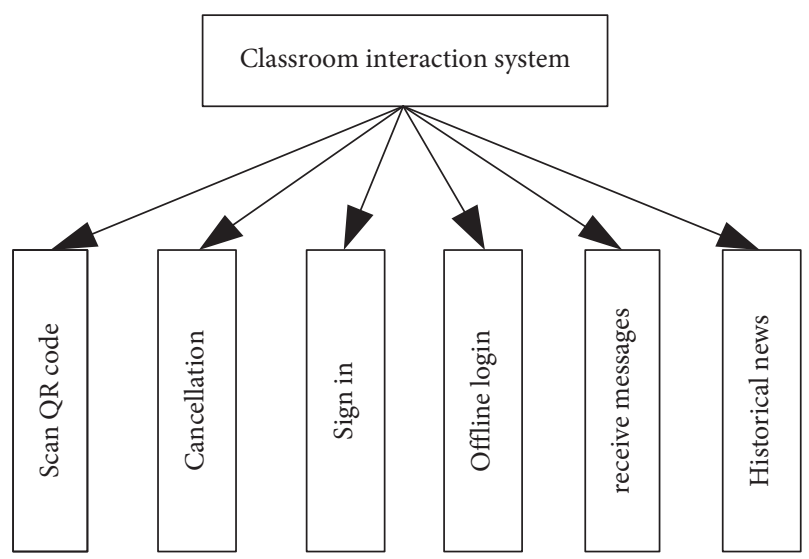

FIgURE 6: Schematic diagram of student interaction module.

processor server is $3.4 \mathrm{GHz}$, and it has $64 \mathrm{~GB}$ of operation space. It has $5 \mathrm{~TB}$ backup storage space for college English teaching data. The data transmission node between English equipment and the Internet is mainly controlled by the network node inside the controller and employs the core controller module of the ARM-7856 signal. The front end is mainly responsible for receiving and sending data, and the back end is used for network perception and adjustment of teaching data in the process of interactive integration. To realize the interactive integration of English teaching data in colleges and universities, a data interactive sensor is applied in the hardware system, which is associated with the web interface. The instructions sent by the web interface can explore the data through the sensor. At the same time, the teaching data collected by the sensor can also be transmitted and reintegrated through the web interface, and the direct interactive control of the teaching data can be further realized by the managers. The core of this sensor is the DIK data-sensitive recognition chip, and the internal circuit of the chip participates in the connection of the data node. Therefore, the chip can accurately identify the data type in the data environment of English teaching in colleges and universities. The circuit diagram of the DIK data-sensitive chip is shown in Figure 9.

The network switch in Figure 10 is a type of network hardware that receives and forwards data to a target device using message switching. The technical parameters of network switches are given in Table 1.

The microprocessor in Figure 10 refers to a central processing unit that is composed of one or several large-scale integrated circuits and has the functions of an arithmetic unit and a controller. It is the core of the system [14].

\subsection{Software Design}

2.2.1. Mining the English Course Learning Resources in the Web Environment. To realize the information sharing of English-assisted instruction systems under the web environment, a model of information detection and resource distributed fusion of English-assisted instruction systems under the web environment is established based on the data mining and feature fusion method of the English-assisted instruction system. The statistical data mining [15] and fuzzy feature detection method [16] are adopted to carry out the decision-making scheduling and adaptive optimization of the information process of English-assisted instruction system.

The information management ability of English-assisted teaching system is improved using subspace clustering 


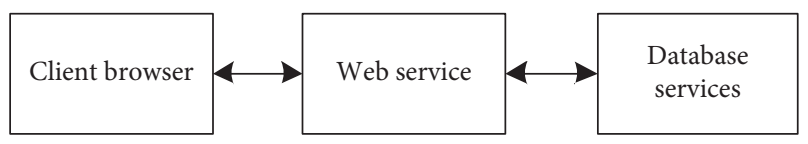

FIgURE 7: Schematic diagram of the logical structure of web server.

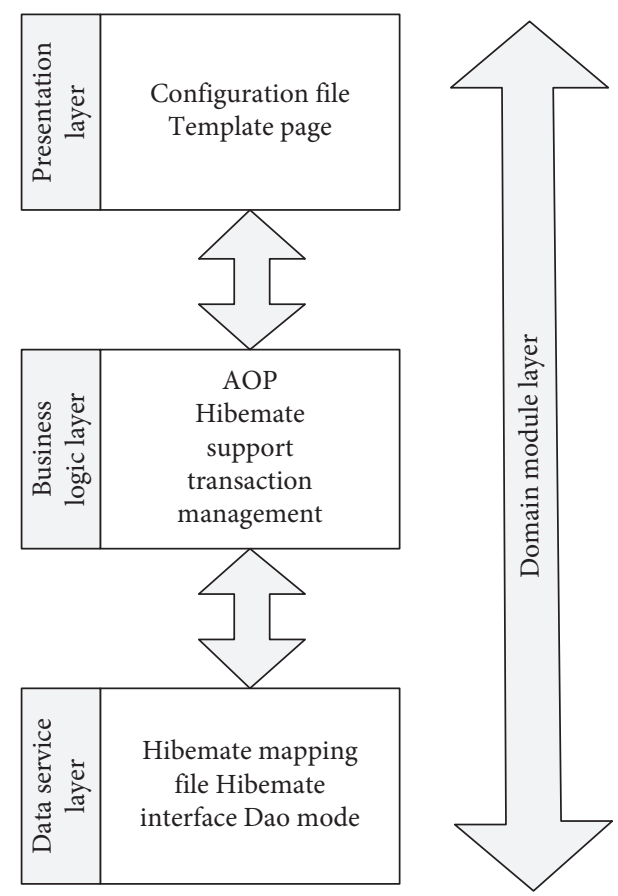

FIGURE 8: Schematic diagram of integrated SSH framework.

analysis, scheduling decision function and the fusion feature analysis, and comprehensive control. The information location optimization function of the English-assisted teaching system in the web environment is computed as

$$
D(o, r)=\sum_{i=1}\left|d\left(o^{i+1}, o^{i}\right)+d\left(r^{i+1}, r^{i}\right)\right|
$$

where $(o, r)$ is the interactive learning information coordinate, $d$ is the optimization coefficient, and $i$ represents the optimization times. Using the method of group sample regression analysis, the association rules of English-aided instruction systems are mined in a web environment [17]. Through statistical analysis and fusion decision, the fitness function of English-aided instruction system information is obtained:

$$
X_{(i+1)}=\frac{p * \mid p \text { best }-D(o, r) \mid}{\ln (1 / u)} .
$$

$u$ is in the range of 0 to 1 and represents the weight vector of the distribution of information resources, $p$ represents the fitness coefficient, and pbest is the association coefficient of information fusion. Using a nonlinear test, a reconstruction model of information fusion of an English-assisted instruction system is obtained [18]. We assume $Q(x, y)$ as a random distribution vector, to get the feature update vector $Q^{\prime}\left(x^{\prime}, y^{\prime}\right)$ and make a comprehensive decision on the information resource management of the English-aided

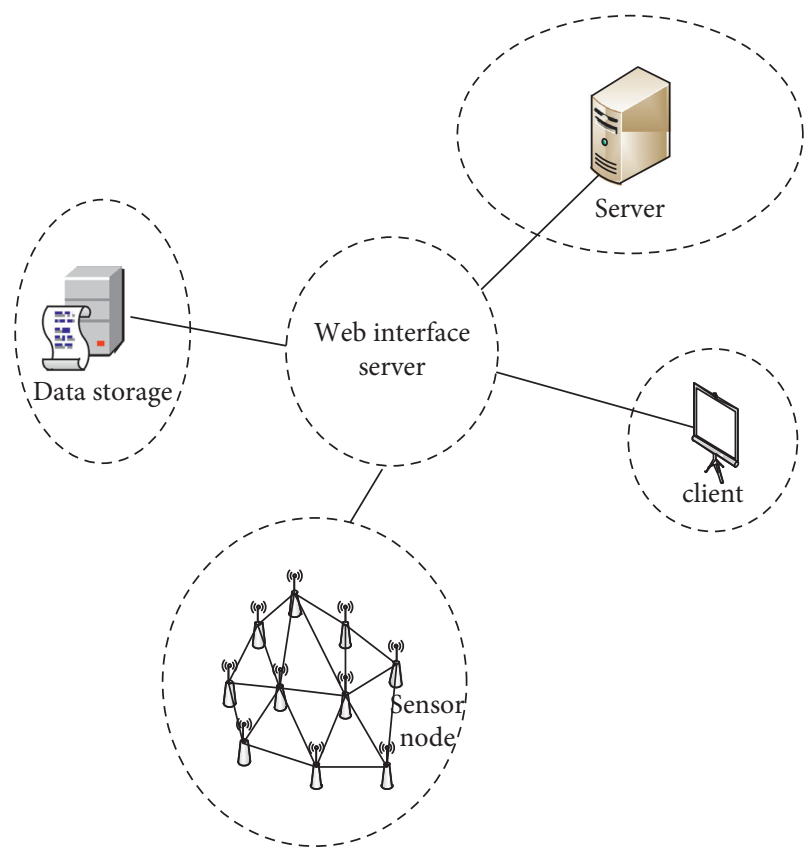

FIgURE 9: Hardware system architecture diagram.

instruction system to get the fitness value of $V_{k}=\left(v_{x}^{k}, v_{y}^{k}\right)$ of the information scheduling of the English-aided instruction system, then the fuzzy parameters of the information scheduling of the English-aided instruction system are $V_{k}=$ $\left(v_{x}^{k}, v_{y}^{k}\right)$ and $y^{\prime}$, and the number of information scheduling of the English-aided instruction system is $k$. The results of sample regression analysis through ruleset fusion are obtained as

$$
\left\{\begin{array}{l}
v_{x}^{k} t=x-k x^{\prime} X_{(i+1)}, \\
v_{y}^{k} t=y-k y^{\prime} X_{(i+1)}
\end{array}\right.
$$

where $t$ is the fusion time. With the help of comprehensive ranking, the information scheduling and spatial parameter ranking of an English-aided teaching system in a web environment are accomplished [19].

$$
W=\min \left\{\sum_{i=1}[\operatorname{STA}(i)+\operatorname{ETA}(i)]\right\}\left(\begin{array}{c}
v_{x}^{k} t \\
v_{y}^{k} t
\end{array}\right) .
$$

Based on the above analysis, in the environment of the ZigBee network, the information sharing and integration scheduling of English-assisted instruction systems are carried out to improve the resource sharing and scheduling ability of English-assisted instruction systems under the web environment [20].

\subsubsection{Data Fusion and Processing of College English Auxiliary} Teaching System. In this study, a fusion model of college English teaching aided resources is constructed which adopts the fusion particle swarm algorithm method [21] to realize the adaptive scheduling of college English teaching aid resources. Moreover, the self-correlation characteristics quantity of the proposed model is extracted and the storage 


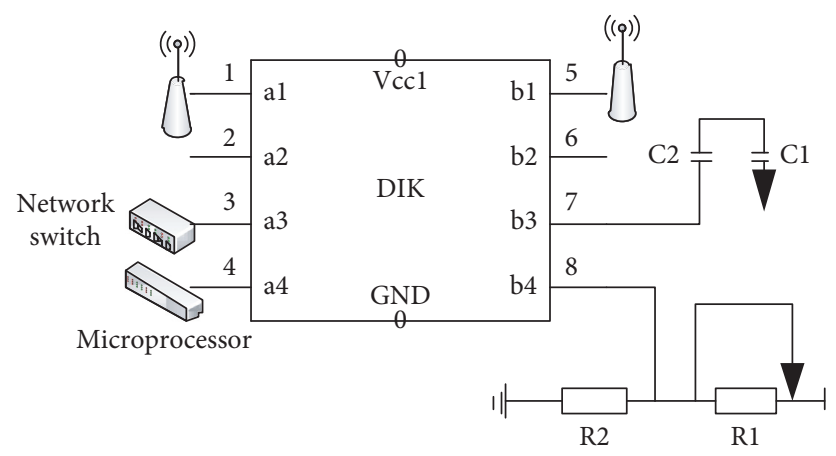

FIgURE 10: Circuit diagram of DIK data-sensitive identification chip.

TABLE 1: Technical specifications of network switches.

\begin{tabular}{lc}
\hline The name of the & Indicators \\
\hline Web standards & IEEE802.3, IEEE8023u, IEEE802.3AB, and \\
IEEE802.3x
\end{tabular}

structure model of the database is obtained. In addition, the template matching and the panel data fusion methods are employed to obtain the data clustering parameter distribution set and construct the dimension reduction model of college English teaching aid system data, which is expressed as follows:

$$
y(t)=x(t) \iint_{\varphi} b(\varphi) \exp [f(t)] \mathrm{d} t \mathrm{~d} \varphi .
$$

$b(\tau, \varphi)$ refers to the spread spectrum parameter of the resource scheduling of the college English-assisted instruction system, $f(t)$ is the steady eigenvalue of the resource distribution, and the semantic window function of the resource scheduling is constructed using feature transformation and fitness training, which is expressed as

$$
H(t)=\frac{N(t)}{y(t)} .
$$

In equation (6), $N(t)$ is the resolution of the associated semantic features of the data of the college English-assisted instruction system. By adopting the association rules detection method of semistructured data, the data fusion function is established [22], and the teaching load balance scheduling model is obtained which is computed using the following equation:

$$
S_{\text {Load balancing }}=H(t)+e^{(f(t)+K / 2)} .
$$

Using fused particle swarm optimization, we get the data mining fused particle swarm scheduling model of college English-assisted teaching system, construct the spatial clustering model of data fusion, and obtain the association rule function of linear scheduling:

$$
G=S_{\text {Load balancing }} \cdot \frac{[(1-\rho) s(t)+\Delta y(t)]}{s(t)} .
$$

The expression $s(t)$ indicates the strength of information transmitted by the system at time $t$. Based on the above analysis, a fuzzy control parameter model is constructed to improve the adaptive coding ability of the data by integrating PSO scheduling.

2.2.3. College English Auxiliary Teaching System Resource Scheduling. Through fuzzy correlation feature matching and statistical analysis, the adaptive optimization of college English auxiliary teaching resource fusion is realized. Combined with statistical feature analytic control and association rule mapping, the fuzzy iterative expression of college English auxiliary teaching resource fusion is obtained as follows:

$$
W=\frac{C e^{(f(t)+K / 2)} p(t)}{e},
$$

where $C$ represents the data fusion characteristic value of college English-assisted teaching system, and the fuzzy state function is obtained by high-dimensional spatial reconstruction:

$$
u=W+\sum_{t}^{-1} v(t)-s(t)
$$

Using the method of semantic abstract expression, the incremental fusion of college English teaching aid is constructed to obtain the iterative function. Under the framework of deep learning, this study extracts the relevant degree information of English teaching resources scheduling and obtains the reliability evaluation function of English teaching information fusion.

$$
Q=u \sum_{i=1} d_{a, i}+d_{b, i}
$$

$d_{a, i}$ and $d_{b, i} \in[1,5]$ represent the related constraint coefficients, construct the resource scheduling model of college English-assisted teaching system, and realize the teaching resource fusion control. 
2.2.4. Reliability Integration of College English Auxiliary Teaching Resources. The underlying database of the college English auxiliary teaching system was constructed and realized in the process of fused particle swarm optimization to schedule college English auxiliary teaching resources. The mathematical description of the fuzzy constraint index distribution problem is as follows:

$$
R=Q \cdot\left[f_{i}(x)-g_{i}(x)\right]
$$

where $f_{i}(x)$ is the level of English teaching resources scheduling, and $g_{i}(x)$ is the conditional cost function of English teaching resources.

The quantitative index system of college English teaching aid is presented as follows:

$$
x_{n}=\frac{a_{0} \sum_{i=1} x_{n-i}+u}{R} .
$$

In (13), $a_{0}$ is the information distribution amplitude of the big data; $x_{n-i}$ is the scalar time series of the distribution of English teaching resources. Using the big data decision tree model, the optimal distribution of online scheduling $\kappa$ of college English teaching assistance resources is obtained as follows:

$$
H=\frac{\kappa}{2}+\sum_{n=1} x_{n}
$$

Using the method of combined density analysis, this paper constructs the conditional probability density parameters of English teaching resources integration:

$$
L=\frac{H}{\left(X_{n}-1\right) \cdot R} \text {. }
$$

According to the joint parameter distribution set of English teaching resource scheduling, the probability density function is computed as

$$
M_{\text {probability density }}=L-f\left(x_{n-1}\right) .
$$

In the process of fused particle swarm optimization, the scheduling of English teaching resources and information fusion is realized, and the optimal control function is expressed as

$$
V_{\text {Control function }}=\frac{M_{\text {probability density }}}{2}+R \cdot \sum_{i=1}^{n}\left(f_{i}(x)\right) \text {. }
$$

Based on this, an adaptive control model of English teaching resource scheduling is obtained, and the system design flow is shown in Figure 11.

\section{Experimental Setup and Validation}

In the course of the experiment, we first determined whether the hardware equipment of the system is in a normal working state and then opened the data transmission mode on each English production equipment. Next, the system was prepared to receive the teaching data of the web interface port from the Internet and the data collection structure was mobilized to collect the characteristics or attributes of the teaching data of college English and plan the expected results of the experiment. The real-time data and offline data were selected as the main data comparison object in the expected results. The experimental setup of this study is depicted in Figure 12.

The hardware equipment gradually inputs the teaching data into the program, inquires the data receiving status in the storage container, and analyzes the data integration parameters. An example diagram of real-time data visualization in network nodes on the web interface is shown in Figure 13.

During the experiment, the intelligent teaching system based on deep learning designed in [3] and the informationbased teaching management system based on the fusion association rule mining algorithm proposed in [4] were used as the control system. Using data visualization techniques, this study compared and analyzed the degree of interaction in all of the three systems, received the same college English teaching data as input, retrieved the interactive data in the three system platforms, and calculated the percentage of the data that can complete the interactive task in all the data. Figure 14 provides a comparison of the degree of interaction for the three systems.

According to the results of the comparison among the three systems, the degree of interaction of the proposed system is the highest, the interactive data that can be realized by the web interface accounts for about $80 \%$ of the total data, while the interactive data in [3] system accounts for about $50 \%$ of the total data, and the interactive data in [4] system accounts for about $40 \%$ of the total data. The proposed system visualized the English Internet data through the design of the visual program in the web interface, so that the managers can directly operate the data and integrate the real-time data and offline data in a corresponding way to create a good interface for the interaction.

3.1. Convergence Contrast. The convergence curve of English-assisted teaching resource sharing of different systems is shown in Figure 15.

The analysis of Figure 15 shows that the convergence error of the proposed method is 0.02 , which is the lowest at 4 min when the initial convergence error is the same. The convergence error of the system given in [3] reaches the lowest value of 0.11 at $9 \mathrm{~min}$, and that in [4] reaches the lowest value of 0.17 at $10.5 \mathrm{~min}$. The above experimental data validate that the proposed method has good convergence in scheduling English-assisted instruction systems in the web environment.

3.2. Comparison of Configuration Accuracy. The accuracy of the configuration of the college English auxiliary teaching system in the web environment by different methods is tested, and the comparison results are shown in Table 2.

Table 2 shows that the accuracy of the systems given in $[3,4]$ is lower than that of the present method in the course of iterative calculation. For example, at iteration 100 the accuracy of the proposed system is $99 \%$ as compared to the other two systems which are $81 \%$ and $85 \%$, respectively. 


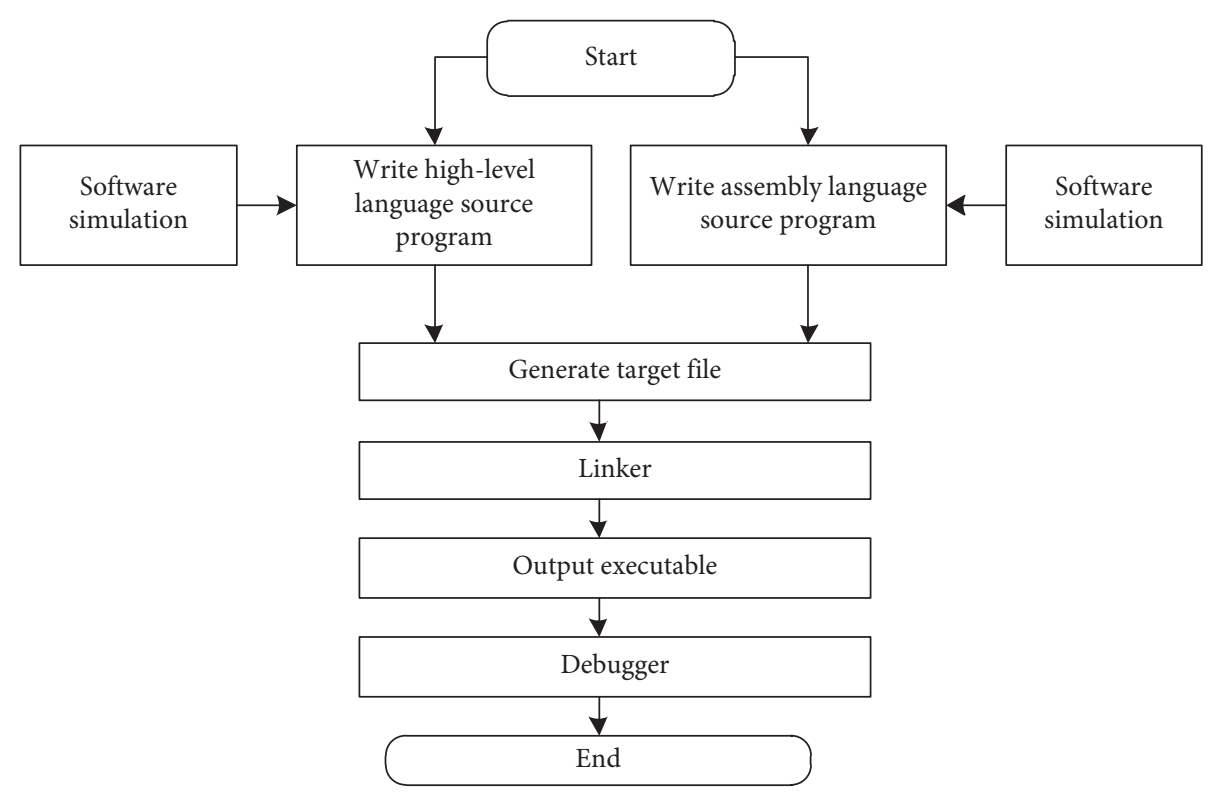

FIgURe 11: Design flow of college English-assisted instruction system.

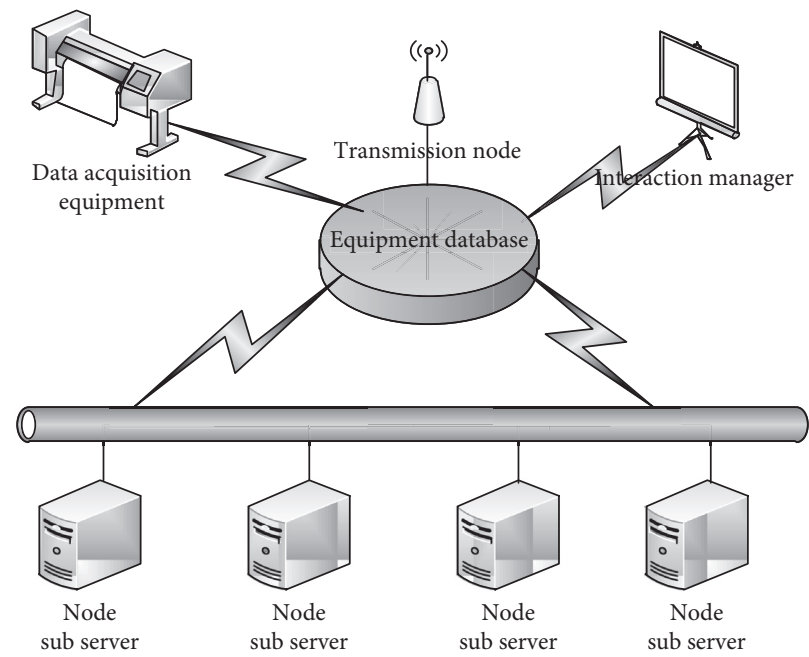

Figure 12: Experimental setup.

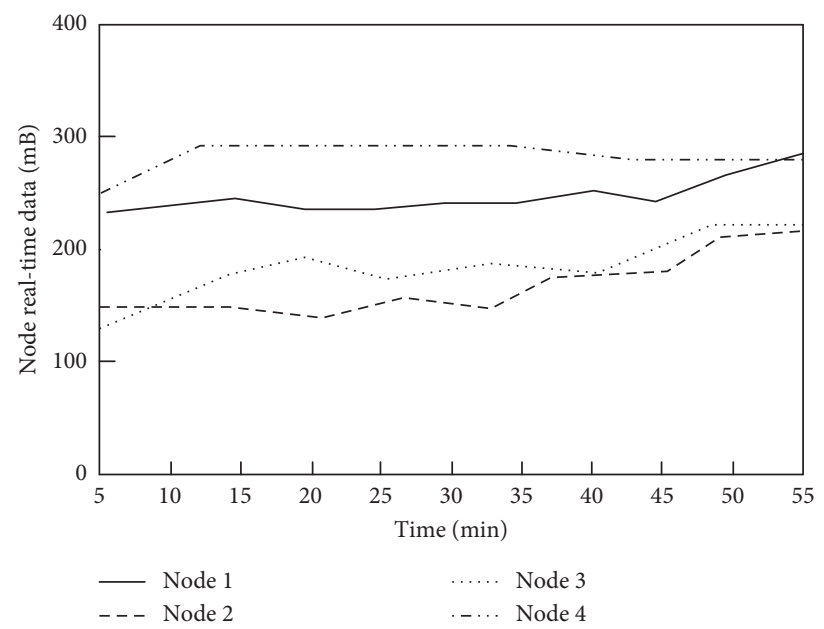

FIgURE 13: Example diagram of real-time data visualization in network nodes on the web interface. 


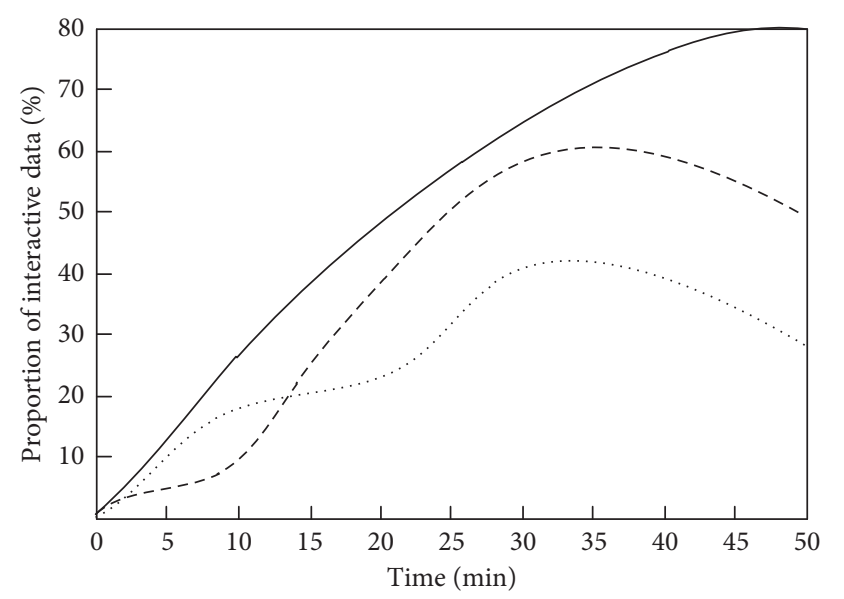

- System designed in this paper

..... Intelligent teaching system based on deep learning - - - Information teaching management system

Figure 14: Comparison of interaction degree of three systems.

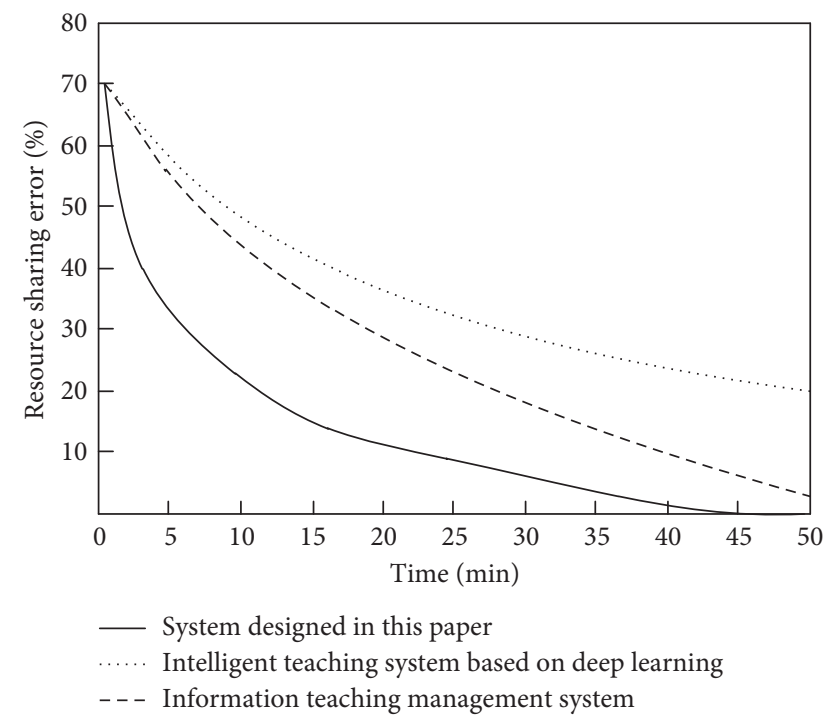

FIGURE 15: Convergence curves of different systems.

TABLE 2: Comparison of configuration accuracy.

\begin{tabular}{lccc}
\hline $\begin{array}{l}\text { Number of } \\
\text { iterations }\end{array}$ & $\begin{array}{c}\text { Proposed } \\
\text { system }\end{array}$ & $\begin{array}{c}\text { Reference } \\
{[3]}\end{array}$ & $\begin{array}{c}\text { Reference } \\
{[4]}\end{array}$ \\
\hline 10 & 0.991 & 0.871 & 0.855 \\
20 & 0.992 & 0.833 & 0.718 \\
30 & 0.991 & 0.885 & 0.773 \\
40 & 0.994 & 0.837 & 0.805 \\
50 & 0.994 & 0.867 & 0.818 \\
60 & 0.996 & 0.813 & 0.890 \\
70 & 0.995 & 0.882 & 0.887 \\
80 & 0.999 & 0.857 & 0805 \\
90 & 0.998 & 0.825 & 0.808 \\
100 & 0.999 & 0.818 & 0.855 \\
\hline
\end{tabular}

Experimental results show that the proposed method can achieve high accuracy in the configuration and scheduling of English-assisted instruction systems in the Web environment. This is because the system is implemented with the help of Android mobile terminal and Web and uses JSON as the data exchange format between the intelligent terminal and the server and designs a Web-based interactive integration module of English teaching data, which realizes the decision scheduling and self-adaptive optimization and completes the mining of English teaching resources.

\section{Conclusion}

With the advent of online learning, the conventional teaching method has changed greatly, progressively changing from teacher-centered to student-centered, with the learner becoming the subject of teaching, and the teacher's role in teaching changing from preacher to instructor. To solve the problem of resource sharing and scheduling in traditional college English-assisted teaching system, a new online college English-assisted teaching system based on the web is presented in this study. Using JSON as the data communication format, the system is implemented using an Android terminal and web server. Data mining and fuzzy feature extraction techniques are used to implement the decision-making scheduling and self-adaptive optimization of English teaching resources in a web aided English teaching system. The particle swarm optimization algorithm is employed to integrate English teaching resources and implement adaptive scheduling. To verify the application performance of the designed system, a simulation experiment is designed and the system was compared with existing systems. The results show that the proposed system is more convergent and more accurate in the allocation and scheduling of instructional resources. The system can improve the overall quality and overall performance of English learning for our society.

\section{Data Availability}

The data used to support the findings of this study are available from the corresponding author upon request.

\section{Conflicts of Interest}

The authors declare that they have no conflicts of interest.

\section{References}

[1] Q. Guo, X. Zhou, and X. Gao, "Research on learning and teaching of languages other than English in system," System, vol. 100, no. 3, Article ID 102541, 2021.

[2] M. Ryshina-Pankova, W. Barthold, and E. Barthold, "Enhancing the content- and language-integrated multiple literacies framework: systemic functional linguistics for teaching regional diversity," System, vol. 96, no. 2, Article ID 102403, 2021.

[3] J. Y. Chen, Z. Wang, and J. Y. Chen, "Design and Research on Intelligent Teaching System Based on Deep Learning," Computer Science, vol. 06, pp. 550-554, 2019.

[4] Q. Zhu, "Design of information teaching management system integrating association rule mining algorithm," Modern Electronics Technique, vol. 43, no. 23, pp. 167-171, 2020. 
[5] H. Zhao and L. Guo, "Design of intelligent computer-aided network teaching system based on web," Computer-Aided Design \& Applications, vol. 19, no. S1, pp. 12-23, 2019.

[6] C. Meng, L. Dan, and W. Jun, "A study of college English culture intelligence-aided teaching system and teaching pattern," English Language Teaching, vol. 13, no. 3, 2020.

[7] F. Aparicio, M. L. Morales-Botello, M. Rubio et al., "Perceptions of the use of intelligent information access systems in university level active learning activities among teachers of biomedical subjects," International Journal of Medical Informatics, vol. 112, pp. 21-33, 2018.

[8] X. Lu, "An empirical study on the artificial intelligence writing evaluation system in China CET," Big Data, vol. 7, no. 2, pp. 121-129, 2018.

[9] C. G. Thompson and S. von Gillern, "Video-game based instruction for vocabulary acquisition with English language learners: a Bayesian meta-analysis," Educational Research Review, vol. 30, no. 12, Article ID 100332, 2020.

[10] D. C. Weng, X. Gan, W. Chen, S. Ji, and Y. Lu, "A new DGNSS positioning infrastructure for android smartphones," Sensors, vol. 20, no. 2, p. 487, 2020.

[11] S. Chen, Y. Zhou, and Y. Chen, "An efficient tool to generate consensus reads for error suppressing and duplicate removing of NGS data," BMC Bioinformatics, vol. 20, no. S23, pp. 1580-1591, 2019.

[12] M. Kulawiak, A. Dawidowicz, and M. E. Pacholczyk, "Analysis of server-side and client-side Web-GIS data processing methods on the example of JTS and JSTS using open data from OSM and geoportal - ScienceDirect," Computers \& Geosciences, vol. 129, pp. 26-37, 2019.

[13] C.-L. Chen, Y.-Y. Deng, W. Weng, M. Zhou, and H. Sun, “A blockchain-based intelligent anti-switch package in tracing logistics system," The Journal of Supercomputing, vol. 77, no. 7, pp. 7791-7832, 2021.

[14] M. M. Parkinson and D. L. Dinsmore, "Understanding the developmental Trajectory of second language acquisition and foreign language teaching and learning using the model of Domain Learning," System, vol. 86, Article ID 102125, 2019.

[15] K. Mahboob, S. A. Ali, and U. Laila, "Investigating learning outcomes in engineering education with data mining," Computer Applications in Engineering Education, vol. 28, no. 6, pp. 76-88, 2020.

[16] A. Arabi, N. Bourouba, A. Belaout, and M. Ayad, "An accurate classifier based on adaptive neuro-fuzzy and features selection techniques for fault classification in analog circuits," Integration, vol. 64, pp. 50-59, 2019.

[17] C. Tjortjis, "Mining Association Rules from Code (MARC) to support legacy software management," Software Quality Journal, vol. 28, no. 6, pp. 458-467, 2020.

[18] J. Feng, X. He, Q. Teng, C. Ren, H. Chen, and Y. Li, "Reconstruction of porous media from extremely limited information using conditional generative adversarial networks," Physical Review, vol. 100, no. 3-1, Article ID 33308, 2019.

[19] Q. Xu, Q. Guo, and C. X. Wang, "Network differentiation: a computational method of pathogenesis diagnosis in traditional Chinese medicine based on systems science," Artificial Intelligence in Medicine, vol. 2021, no. 7724, Article ID 102134, 2021.

[20] L. Minmin, H. Jiang, H. Yule et al., "A systematic review on botany, processing, application, phytochemistry and pharmacological action of Radix Rehmnniae," Journal of Ethnopharmacology, vol. 285, Article ID 114820, 2021.

[21] G. F. Templeton, M. Kang, and N. Tahmasbi, "Regression imputation optimizing sample size and emulation: demonstrations and comparisons to prominent methods," Decision Support Systems, vol. 151, no. 3, Article ID 113624, 2021.

[22] R. M. Adnan, R. R. Mostafa, O. Kisi, Z. M. Yaseen, S. Shahid, and M. Zounemat-Kermani, "Improving streamflow prediction using a new hybrid ELM model combined with hybrid particle swarm optimization and grey wolf optimization," Knowledge-Based Systems, vol. 230, no. 6, Article ID 107379, 2021. 